\title{
Fluorescent Labeling Study of Plasminogen Concentration and Location in Simulated Bovine Milk Systems
}

\author{
L. Wang, K. D. Hayes, and L. J. Mauer ${ }^{1}$ \\ Department of Food Science, Purdue University, West Lafayette, IN 47907
}

\begin{abstract}
A fluorescent labeling method was developed to study plasminogen (PG) concentration and location in simulated bovine milk. Activity and stability of PG labeled with Alexa Fluor 594 (PG-594) were comparable to those of native PG. The fluorescent signal of PG-594 exhibited $\mathrm{pH}$, temperature, and storage stability, and remained stable throughout typical sample treatments (stirring, heating, and ultracentrifugation). These characteristics indicate broad applicability of the fluorescent labeling technique for milk protease characterization. In an example application, PG-594 was added to simulated milk samples to study effects of heat and $\beta$ lactoglobulin ( $\beta$-LG) on the distribution of PG. Before heating, about one-third of the PG-594 remained soluble in the whey fraction (supernatant) whereas the rest became associated with the casein micelle. Addition of $\beta$-LG to the system slightly shifted PG-594 distribution toward the whey fraction. Heat-induced PG-594 binding to micelles in whey-protein-free systems was evidenced by a decrease of PG-594 from 31 to $15 \%$ in the whey fraction accompanied by an increase of PG-594 from 69 to $85 \%$ in casein micelle fractions. When $\beta$-LG was present during heating, more than $95 \%$ of PG-594 became associated with the micelle. A comparison with the distribution pattern of PG-derived activities revealed that heat-induced $P G$ binding to micelles accompanies heat-induced PG inactivation in the micelle fraction. Incubation of the casein micelles with the reducing agent $\beta$-mercaptoethanol revealed that disulfide bonds formed between PG and casein or between PG and casein-bound $\beta$-LG are the mechanisms for heat-induced PG binding to casein micelles. Western blotting and zymography results correlated well with fluorescent labeling studies and activity studies, respectively. Theoretically important findings are: 1 ) when heated, serum PG is capable of covalently binding to micellar casein or complexing with $\beta$-LG in whey and then coadhering to micelles, and 2) PG that associated with micellar
\end{abstract}

Received July 1, 2005.

Accepted September 1, 2005.

${ }^{1}$ Corresponding author: mauer@purdue.edu casein through lysine binding sites before heating is capable of developing heat-induced disulfide bonds with casein. The overall results are PG covalently binding to micelles and inactivation thereafter. Our results suggest that, instead of thermal denaturation through irreversible unfolding, covalent bond formation between PG and other milk proteins is the mechanism of $P G$ inhibition during thermal processing.

Key words: fluorescent labeling, plasminogen, bovine milk, $\beta$-lactoglobulin

\section{INTRODUCTION}

Plasmin (PL; EC 3.4.21.7) is the major native protease found in milk. It readily hydrolyzes $\beta$-casein, $\alpha_{\mathrm{S}^{-}}$ casein, and (more slowly) $\alpha_{\mathrm{S} 1}$-casein (Fox, 1989). Plasmin plays an important role in flavor development and texture changes during cheese ripening (Bastian and Brown, 1996; Fox and McSweeney, 1996) and in influencing stability of UHT milk (Enright et al., 1999; Scollard et al., 2000). Plasmin is primarily secreted in milk as plasminogen (PG), its inactive (zymogen) form, and activated during storage by plasminogen activators (PA; Derham and Andrews, 1982). Plasmin activity and PG activation can be inhibited by plasmin inhibitors and plasmin activator inhibitors. However, these inhibitors do not significantly affect commercial dairy products because they are readily inactivated by pasteurization or acid treatment of milk (Nielsen, 2002).

In fresh milk, the majority of PL and PG are associated with casein micelles (Korycka-Dahl et al., 1983; Politis et al., 1992). Plasmin and PG can be dissociated from the casein micelle by the addition of either $\varepsilon$ aminocaproic acid (EACA) or $\mathrm{NaCl}$, or by acidification of the milk, suggesting that lysine binding as well as electrostatic forces are involved in PL/PG binding to casein micelles (Grufferty and Fox, 1988a). A number of dairy processing conditions, such as $\mathrm{pH}$ and temperature, and growth of psychrotrophic bacteria might affect PL/PG association with casein micelles (Benfeldt et al., 1995; Fajardo-Lira et al., 2000; Crudden and Kelly, 2003).

Heat treatment of milk increases cheese yield by incorporation of denatured whey proteins into the curd (Swaisgood, 1996). Although increasing cheese yield is 
economically desirable, researchers have shown that the binding of denatured whey proteins, mainly $\beta$-LG, to casein micelles reduces the proteolysis and flavor development during cheese ripening presumably by steric hindrance to $\mathrm{PL} / \mathrm{PG}$ binding to the same casein micelle (Benfeldt et al., 1997; Enright and Kelly, 1999; Kelly, 1999). Additionally, release of PL and PG into the whey fraction of milk could adversely affect the quality and application of whey protein ingredients (de Wit, 1998). Therefore, it is important to characterize the distribution of PG influenced by dairy processing conditions.

When whole milk is heated, $\beta$-LG interacts not only with casein micelles, but also with PL and PG through thiol-disulfide exchange reactions (Alichanidis et al., 1986; Grufferty and Fox, 1986). Plasmin/PG inactivation kinetics due to reactions with $\beta$-LG have been characterized in milk and model systems (Rollema and Poll, 1986; Bastian et al., 1993; Metwalli et al., 1998; Saint Denis et al., 2001b). Studies showed that denatured $\beta$ LG caused PL inhibition starting at $45^{\circ} \mathrm{C}$, whereas heat-induced PL inactivation occurred above $65^{\circ} \mathrm{C}$ (Metwalli et al., 1998). Casein micelles protect PL from heat inactivation and partially compensate for the inhibitory effect of $\beta$-LG (Alichanidis et al., 1986; Rollema and Poll, 1986; Grufferty and Fox, 1988b; Metwalli et al., 1998). Effects of heat and $\beta$-LG on the distribution of PG-derived activity in a milk model system were also investigated (Sombers and Hayes, 2005). However, PGderived activities in both whey and casein fractions diminished after heating, and the effect of heat treatment on the distribution (location and concentration) of PG remains unknown in bovine milk systems.

The majority of published studies on properties and behaviors of PL and PG in bovine milk are based on activity measurements. Plasmin is often analyzed using a specific synthetic substrate, and PL activity is defined as the release of chromogenic (Rollema et al., 1983) or fluorogenic (Richardson and Pearce, 1981) products per unit time. To investigate PG, it is activated to PL by PA and then derived PL activity is measured. All of these activity assays have many limitations such as interference by whey proteins (Politis et al., 1993; Hayes et al., 2002) and competitive inhibition by caseins (Bastian et al., 1991). Therefore, multiple steps of sample preparation are often needed to remove casein and whey protein interferences before activity determination. Each of these sample clean-up steps potentially alters the location and activity of PL and PG components in milk. This often leads to underestimation of PL activity, and it has been reported that only a fraction of PL activity can be recovered in spiked milk (milk with known amounts of added PL) or milk model systems (Choi et al., 2004). Although the sensitivity and detection levels for PL and PG can be improved (SaintDenis et al., 2001a), activity-based assays are inherently unable to distinguish activity from concentration of PL system components, and both pieces of information are extremely important for studying the complexity of the PL system. For example, a change in PL activity can result from changes in PA activity, changes in PG availability, changes in PL activity due to inhibitors, or PL inactivation/denaturation by treatments. Using only an activity measurement is inadequate to explain which of these mechanisms affects the activity. To complement the information gathered using activity assays, a concentration assay able to accurately measurement PG/PL/PA concentrations would be useful to gain a better understanding of the effects of processing treatments on PL system components.

Several ELISA methods have been developed to measure PL and PG concentrations in milk and cheese samples (Politis et al., 1992; Baer et al., 1994; Benfeldt et al., 1995; Dupont et al., 1998). However, application of ELISA is limited by the lack of availability of high quality monoclonal antibodies that differentiate PL from PG. An ELISA method also requires sample clean up to remove the casein inhibitory effect before measurement (Dupont and Grappin, 1998), and the cleanup steps result in limited accuracy. Other methods, including zymography (Lu and Nielsen, 1993) and Western blotting (Politis et al., 1992; Benfeldt et al., 1995), have been used to visualize the PL proteins. However, these methods lack the high throughput and accuracy needed for quantitative studies. Therefore, none of the current methods is capable of accurately measuring the concentration of plasmin system components in the presence of casein.

Labeling proteins with fluorophores, affinity tags, or other chemical or optically active species is widely used for studying protein functions in living cells or tissues. The ease of visualization by fluorescent microscopy and quantification of fluorescently labeled proteins by spectrofluorometric measurement has led to the widespread application of fluorescent-labeling methods and has enabled researchers to locate, track, and quantify proteins of interest in highly complex biological systems (Chamberlain and Hahn, 2000; Miller and Cornish, 2005). Since the initial description of the use of fluorescently labeled antibodies in tissue sections (Coons, 1951), a variety of fluorescent probes have been developed and many are commercially available. Some of fluorescent dyes such as fluorescin, cyanine, or Alexa have minimum impact on the biological properties of the protein and the labeling process is straightforward (Holmes and Lantz, 2001). When combined with subcellular fractionation, fluorescent labeling of proteins has been successfully used to define protein redistribution in re- 
sponse to a variety of physiological stimuli (Patton, 1999). Additionally, the possibility of direct visualization of protein-protein interactions by fluorescent resonance energy transfer (Kenworthy, 2001; Centonze et al., 2003; Herman et al., 2004) makes fluorescent labeling an ideal research tool for investigating distributions and interactions of plasmin system components in milk.

Milk is one of the most complex biological systems. Lack of an effective method to characterize plasmin system components in the presence of casein has hindered the understanding of the plasmin system and the ability to control the plasmin system during dairy processing. Because PG exists in much higher concentrations than PL in milk and is the major source of proteolytic activity, the objectives of this research were to develop a novel concentration-based fluorescent-labeling method to study PG, and to use this method to examine the distribution pattern of $\mathrm{PG}$ in a simulated bovine milk system influenced by heat-induced $\beta$-LG binding to casein micelles.

\section{MATERIALS AND METHODS}

\section{Reagents}

The following chemicals were obtained from the indicated sources: Tris base, sodium chloride, potassium citrate, trisodium citrate, magnesium citrate, Tween 80, EACA, and gelatin were purchased from SigmaAldrich Corp. (St. Louis, MO). Potassium phosphate monobasic, potassium sulfate, potassium hydroxide, potassium chloride, calcium chloride, and potassium carbonate were obtained from Mallinckrodt Baker Inc. (Paris, KY). Nitrocellulose membrane, Laemmli sample buffer, and $\beta$-mercaptoethanol (2ME) were purchased from BioRad Laboratories (Richmond, CA).

\section{Sources of Milk Proteins and Enzymes}

Bovine milk was drawn from the bulk tank at the Purdue University Dairy Center (West Lafayette, IN). Fresh whole milk samples were sent to Universal Laboratory Services (Lansing, MI) for protein, fat, somatic cell, solid, and lactose analysis to ensure quality of the milk. The milk was immediately skimmed at 5,000 $\times \mathrm{g}$ for $20 \mathrm{~min}$ at $4^{\circ} \mathrm{C}$ and used for micelle isolation by ultracentrifugation at $90,000 \times g$ for $1 \mathrm{~h}$ at $4^{\circ} \mathrm{C}$ in a Beckman L7 ultracentrifuge and associated sw-28 rotor (Beckman Coulter Inc., Fullerton CA). The experimental casein micelle samples were prepared first by grinding the casein pellet to a homogeneous suspension using a pestle and mortar and then reconstituting to the original milk volume in synthetic milk salts buffer (JKB) described by Jenness and Koops (1962). The reconstituted micelle samples were stirred for $20 \mathrm{~h}$ at $4^{\circ} \mathrm{C}$ before further treatment. Tween $80(0.01 \% \mathrm{vol} / \mathrm{vol})$ was added to the samples as a stabilizer.

Native $\beta$-LG was obtained from Davisco Foods International, Inc. (Eden Prairie, MN). Bovine PG and high molecular weight human urokinase (uPA) were obtained from American Diagnostica Inc. (Stamford, CT). Plasminogen was used for fluorescent modification and served as an activity and electrophoresis standard. Bovine PL was purchased from Roche Diagnostics Co. (Indianapolis, IN) and used as the standard control in protein electrophoresis.

\section{Fluorescent Labeling of PG and Fluorescent Measurement}

Bovine PG was labeled with Alexa Fluor 594 reactive dye (Molecular Probes, Inc., Eugene, OR). Labeling reactions were carried out according to the manufacturer's instructions with minor modifications. Tween 80 was added to the labeling reaction mixture to a final concentration of $0.01 \%$ ( $\mathrm{vol} / \mathrm{vol}$ ) to increase PG stability. The total reaction mixture was transferred to a BioGel P-30 fine size exclusion purification column and modified Tris buffer (0.05 $M$ Tris, $0.1 M \mathrm{NaCl}$, pH 7.6) was used as an elution buffer to separate PG-Alexa Fluor 594 conjugate (PG-594) from the free dye. Purified PG594 was quantified using a bicinchinonic acid (BCA) protein assay kit (Pierce, Rockford IL) and adjusted to $1 \mathrm{mg} / \mathrm{mL}$ in modified Tris buffer. Activity of labeled PG was measured in triplicate using the chromogenic substrate Spectrozyme PL in the presence of uPA (Fajardo-Lira et al., 2000).

To measure fluorescence of diluted PG-594 in buffer, two $200-\mu \mathrm{L}$ fractions of each sample were transferred to a 96-well costar black plate (Corning, NY). Fluorescent emission in relative fluorescent units (RFU) at $625 \mathrm{~nm}$ was measured with excitation at $580 \mathrm{~nm}$ and a cutoff filter of $610 \mathrm{~nm}$ using a Flex Station II fluorescent microplate reader (Molecular Devices, Sunnyvale, CA). For samples containing milk proteins, trisodium citrate solution $(1 M)$ was added at a 1:9 ( $\mathrm{vol} / \mathrm{vol})$ ratio to dissociate micellar casein and reduce sample turbidity for fluorescence measurement.

\section{Fluorescent Stability of PG-594}

To examine temperature effects on the fluorescent stability of PG-594, the PG-594 was diluted in $400 \mu \mathrm{L}$ of JKB to $4 \mu \mathrm{g} / \mathrm{mL}$ and heated in a water bath to $90^{\circ} \mathrm{C}$ for 2 min. For pH stability, PG-594 was diluted to 4 $\mu \mathrm{g} / \mathrm{mL}$ in $400 \mu \mathrm{L}$ of $\mathrm{pH}$-modified JKB ranging from $\mathrm{pH}$ 4 to 8 and fluorescence of the preparation was monitored at room temperature up to $2 \mathrm{~h}$. The storage stability of PG-594 dilutions in JKB was monitored at $4^{\circ} \mathrm{C}$ 
for up to $7 \mathrm{~d}$ and at $-20^{\circ} \mathrm{C}$ for up to $8 \mathrm{mo}$. This experiment was done in duplicate.

The PG-Alexa Fluor 594 conjugate was added to simulated milk systems (reconstituted fresh micelle containing $0.5 \% \beta$-LG) at $2.8 \mu \mathrm{g} / \mathrm{mL}$. Samples were either acid-precipitated or ultracentrifuged to separate casein micelles from the whey fraction (supernatant). Precipitated casein micelles were reconstituted in JKB to the original sample volume. The sum of fluorescent intensity of both whey and micelle fractions was compared with the fluorescent intensity of the untreated samples to evaluate PG-594 fluorescent stability during dairy processing.

\section{Calibration of PG-594 Level Using a Standard Curve}

The effect of milk proteins on fluorescent emission of PG-594 was examined by diluting PG-594 into $400 \mu \mathrm{L}$ of $0.5 \% \beta$-LG or into $3 \%$ casein micelle solution in JKB at concentrations from 0.125 to $4 \mu \mathrm{g} / \mathrm{mL}$. A serial dilution of PG-594 was also heated in a $90^{\circ} \mathrm{C}$ water bath for approximately $1.5 \mathrm{~min}$ to raise the sample temperature above $85^{\circ} \mathrm{C}$ for $16 \mathrm{~s}$. Fluorescence of each sample was measured. Triplicate samples of each dilution were used to construct standard curves for calibration of PG594 levels in whey and casein micelle fractions.

\section{Effect of Heat Treatment on PG-594 (PG-594 Test)}

To investigate the effect of heat treatment and heatinduced $\beta$-LG binding to casein micelles on distribution of PG-594, a factorial design was used to examine effects of fresh micelle sources and 2 treatment factors: levels of $\beta$-LG ( 0 or $0.5 \%)$ and levels of heat treatment (no heat or $86^{\circ} \mathrm{C}$ for $15 \mathrm{~s}$ ). Experiments were conducted in duplicate for each of the 3 different fresh micelle sources.

$\beta$-Lactoglobulin and PG-594 were added directly to $12 \mathrm{~mL}$ of reconstituted casein micelle samples at $0.5 \%$ (wt/vol) and $2.8 \mu \mathrm{g} / \mathrm{mL}$, respectively. These levels are consistent with reported concentrations found in milk (Fox and McSweeney, 2003). The milk protein mixture was allowed to stir for $1 \mathrm{~h}$ at $22^{\circ} \mathrm{C}$ before heat treatment. The sample mixture was submerged in a $90^{\circ} \mathrm{C}$ water bath while under continuous mixing, and the temperature of the sample mixture was monitored using an Omega digital thermometer (model 871, Omega Engineering, Inc., Stamford, CT) and allowed to increase above $85^{\circ} \mathrm{C}$ for $16 \mathrm{~s}$. The total sample heating time was about $6 \mathrm{~min}$. Samples were immediately transferred to an ice water bath after heating. The unheated control samples were kept on ice. Both heated and unheated control samples were fractionated into whey and curd fractions using ultracentrifugation at $60,000 \times g$ for 1 h at $4^{\circ} \mathrm{C}$ in a Beckman L7 ultracentrifuge and associated Ti 70.1 rotor. The centrifugation speed used was experimentally determined as the minimum required to effectively deposit casein micelles as a firm pellet while avoiding sedimentation of soluble $\beta$-LG proteins. Precipitated caseins were further resuspended in JKB to the original sample volume to obtain the micelle fraction.

Concentrations of PG-594 levels in both whey and micelle fractions were determined by fluorescent measurement as described above. Total protein concentration in whey and micelle fractions was determined using BCA.

\section{PG-594 Dissociation from Micelle}

To evaluate the ability of EACA to dissociate PG594 from casein micelles, EACA was added to micelle fractions to a final concentration of $0.25 \mathrm{M}$. The mixture was incubated for $2 \mathrm{~h}$ at $22^{\circ} \mathrm{C}$ before acid precipitation to separate the supernatant from the micelle pellet. To evaluate the ability of a reducing agent to dissociate PG-594 from casein micelles, 2ME was added to micelle fractions to a final concentration of $0.48 \mathrm{M}$. This mixture was also incubated for $2 \mathrm{~h}$ at $22^{\circ} \mathrm{C}$ and ultracentrifugation was used instead of acid precipitation to separate the supernatant from the micelle pellet. Fluorescent measurement of the supernatant was done to evaluate the amount of PG-594 dissociated from casein micelles by either EACA or $2 \mathrm{ME}$ treatments.

\section{SDS-PAGE and Zymography}

Samples of whey and micellar fractions were dissolved on SDS-PAGE to examine how milk protein composition was affected by heat treatments. For electrophoresis under reducing conditions, samples were mixed with Laemmli sample buffer with $2 \mathrm{ME}$ and boiled for $5 \mathrm{~min}$ before loading onto the gel. Electrophoresis was performed using $12.5 \%$ Criterion precast gel systems and prestained broad-range standards (BioRad Laboratories).

For zymography to visualize PG-derived PL activity in micellar fractions, handcast casein gels containing $280 \mathrm{IU} / \mathrm{mL}$ of uPA were used. Micellar samples were analyzed under nonreducing conditions (addition of $2 \mathrm{ME}$ to Laemmli sample buffer and heating of the samples were omitted). After electrophoresis, the gel was washed with $2.5 \%$ Triton $\mathrm{X}-100$ for $1 \mathrm{~h}$ and incubated in modified Tris buffer for $28 \mathrm{~h}$ at $37^{\circ} \mathrm{C}$, followed by Coomassie staining and destaining to visualize lysis zones on the gel as described by Lu and Nielsen (1993). 


\section{Western Blotting}

Proteins in whey and micellar fractions were subjected to SDS-PAGE under both nonreducing and reducing conditions. After electrophoresis, proteins were transferred by electroblotting to a nitrocellulose membrane using a Criterion transblot electrophoretic cell (BioRad). The nitrocellulose membranes were blocked in PBS/Tween buffer containing 3\% (wt/vol) gelatin overnight at $4^{\circ} \mathrm{C}$, and probed with polyclonal goat antibovine PG (American Diagnostics, Greenwich, CT) at 1:2000 dilution in PBS/Tween for $2 \mathrm{~h}$ at room temperature. Further incubation with rabbit antigoat IgGhorseradish peroxidase conjugate (Jackson Immuno Research Laboratories Inc., West Grove, PA) at 1:5000 dilution in PBS/Tween and enhanced chemiluminescence substrate system (Amersham Biosciences Corp, Piscataway, NJ) facilitated signal detection. Autoradiography was performed to Optimum x-ray films (Life Science Products, Frederick, CO).

\section{Fluorescent Confocal Microscopy}

Confocal laser scanning microscopy experiments were conducted on unfractionated samples using a BioRad MRC-1024 laser-scanning confocal attachment (BioRad Laboratories, Hemel Hempstead, UK) mounted on a Nikon Diaphot 300 inverted microscope (Nikon, Tokyo, Japan). The confocal system was equipped with a fluor oil $(60 \times)$ objective lens, an aircooled krypton-argon (568 nm) laser (American Laser, Salt Lake City, UT), a 585-nm long-pass filter, 3 fluorescence detection channels (photomultipliers), and a nonconfocal transmitted-light detector. Image volumes were collected by scanning a series of images, with focus control provided by a precision microstepping motor.

\section{Statistical Analyses}

The effects of heat treatment (no heat or $85^{\circ} \mathrm{C}$ for 16 s), $\beta$-LG ( 0 or $0.5 \%$ levels), and 3 fresh micelle sources on the PG-594 distribution were examined in a $2 \times 2 \times$ 3 factorial model by ANOVA using the SAS program (SAS Institute Inc., Cary, NC). The means for each treatment were separated by Duncan's multirange comparison at a significance level of $\alpha=0.05$.

\section{RESULTS AND DISCUSSION}

\section{Fluorescently Labeled PG (PG-594)}

Alexa fluor 594 dye was experimentally chosen for PG labeling because it is highly fluorescent, heat stable, $\mathrm{pH}$ inert, and photostable. Most importantly, with an

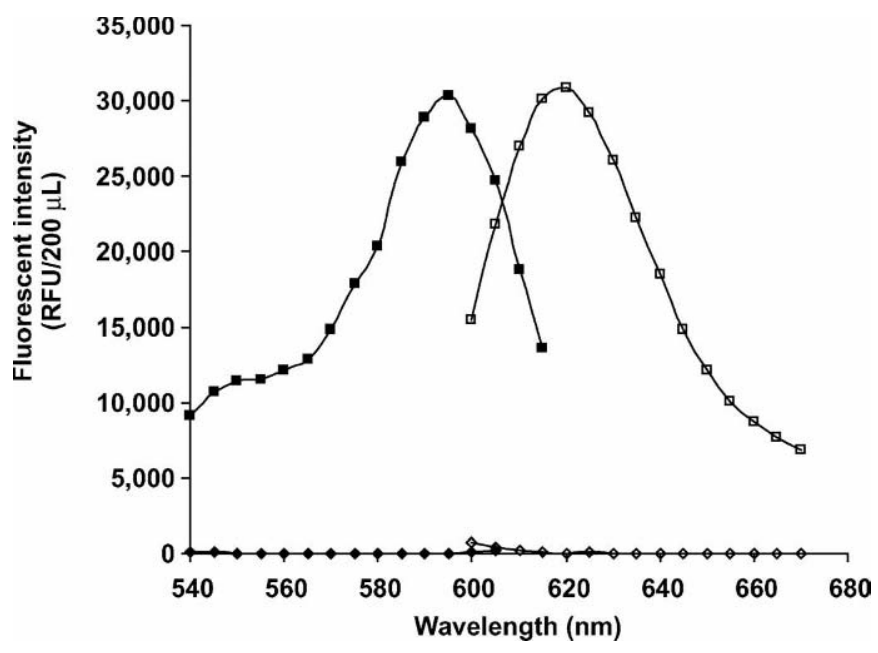

Figure 1. Absorption and fluorescence emission spectra of Alexa Fluor 594 conjugated bovine plasminogen (PG-594) spiked skim milk $(\square, \square)$ and skim milk control $(\diamond, \diamond)$; PG-594 has an absorption maximum at $590 \mathrm{~nm}$ and a red fluorescence emission maximum at $620 \mathrm{~nm} . \mathrm{RFU}=$ Relative fluorescence units.

emission maximum at $625 \mathrm{~nm}$ (Figure 1), fluorescent detection of PG-594 in bovine milk was not affected by native milk fluorophores (Dufour and Riaublanc, 1997; Kulmyrzaev and Dufour, 2002).

The fluorescent signal of PG-594 in buffers exhibited broad range stability in $\mathrm{pH}$ (4 to 8 ), temperature (4 to $90^{\circ} \mathrm{C}$ ), and storage (up to $7 \mathrm{~d}$ at $4^{\circ} \mathrm{C}$ and $8 \mathrm{mo}$ at $-20^{\circ} \mathrm{C}$ ). The signal remained stable throughout typical sample treatments, such as stirring (300 rpm for $12 \mathrm{~h}$ ), acid precipitation ( $\mathrm{pH} 4.6)$, and ultracentrifugation $(90,000$ $\times g$ for $1 \mathrm{~h}$ ) in simulated milk systems.

Activity and stability of PG-594 were comparable to native PG (Figure 2). Both native PG and PG-594 could be activated by uPA; and derived PL activity increased linearly as PG and PG-594 levels increased. However, the slope of activity curve of PG-594 (0.142 \pm 0.008$)$ was lower than that of native PG $(0.185 \pm 0.002)$. This indicates that the fluorescent labeling may have resulted in a decreased affinity in either uPA to labeled PG or derived PL to the synthetic substrate.

The fluorescent intensity increased linearly $\left(\mathrm{R}^{2}>\right.$ 0.999 ) as concentration of PG-594 increased from 0.125 to $4 \mu \mathrm{g} / \mathrm{mL}$ (Figure 3 ). Most importantly, the presence of milk proteins, such as $\beta$-LG and casein micelles, at levels typically found in milk had no significant effect $(P=0.96)$ on the fluorescent intensity of PG-594, demonstrating the potential of using this fluorescent labeling method to quantify PG in the presence of milk casein micelles. This finding indicates that the developed fluorescent techniques could be more useful than ELISA techniques, which require multiple clean-up steps to remove casein proteins before measurement. 


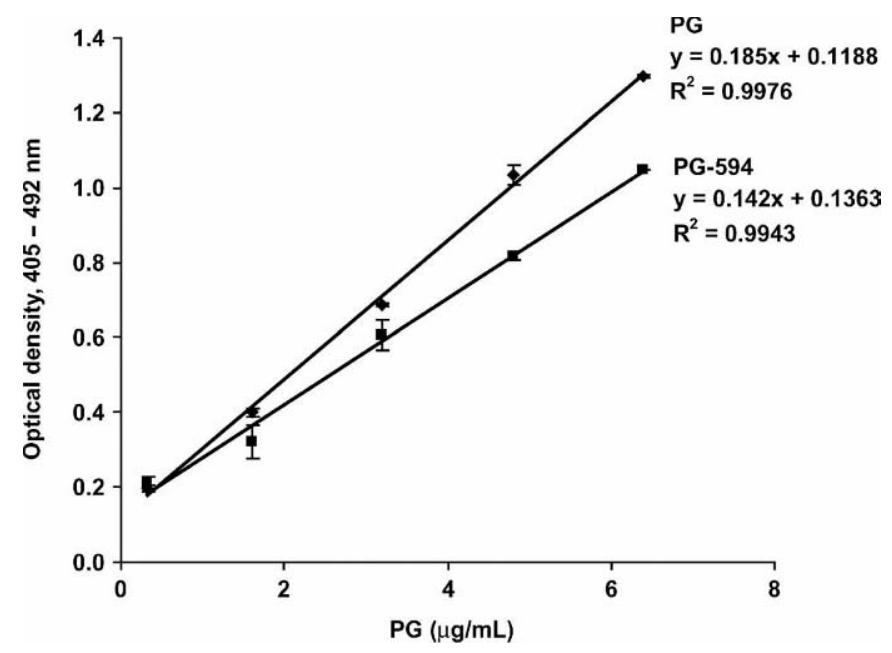

Figure 2. Derived plasmin activity of plasminogen $(\mathrm{PG}, \bullet)$ and Alexa Fluor 594 conjugated plasminogen (PG-594, $\mathbf{0})$ by urokinase in modified Tris buffer. Values are means of 3 determinations, with error bars representing standard deviation.

The effect of a heat-treatment, cream pasteurization $\left(85^{\circ} \mathrm{C}\right.$ for $16 \mathrm{~s}$ ), on PG-594 was also characterized. Results show that although heating had little effect on PG-594 fluorescent intensity in the micelle system $(P=$ 0.06), the slope of the PG-594 standard curve decreased significantly $(P<0.01)$ when $\beta$-LG was present (Figure 3 ). Changes in fluorescent intensity and shifts of maxi-

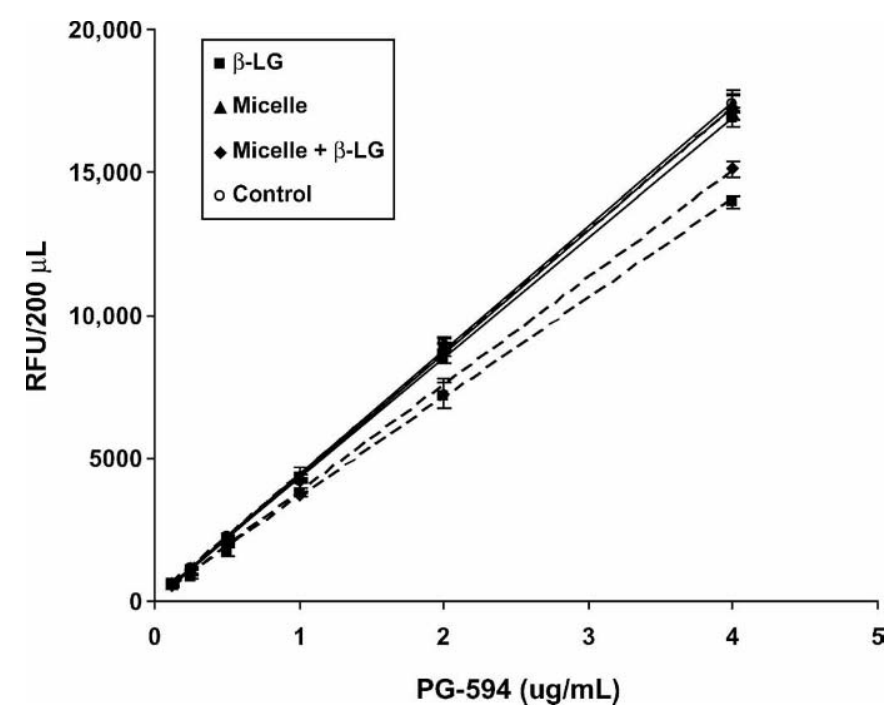

Figure 3. The Alexa Fluor 594 conjugated plasminogen (PG-594) standard curves. The solid curves represent PG-594 in control buffer JKB $(\bigcirc)$ and milk model systems containing casein micelle $(\mathbf{\Lambda}), \beta$ lactoglobulin $(\beta$-LG, $\boldsymbol{\square})$, and both casein and $\beta$-LG $(\bullet)$; Dashed curves represent PG-594 standard curves affected by heat treatment $\left(85^{\circ} \mathrm{C}\right.$ for $16 \mathrm{~s}$ ). Values are means of 3 determinations, with error bars representing standard deviation. All standard curves are linear with $\mathrm{R}^{2}>0.999$. RFU $=$ Relative fluorescence units. mum emission wavelength can occur because of changes in the environment around the fluorophore, such as protein unfolding or protein-protein interactions (Lakowicz, 1999). Compared with unheated standard curves, the reduction of fluorescent intensity indicated that interactions between PG-594 and $\beta$-LG must have changed during heating. Quantification of PG-594 exposed to different heat treatments or different milk system components, therefore, would require different standard curves. In systems without $\beta$-LG or in unheated systems, PG-594 can be evaluated in all fractions of milk using the standard curve developed in JKB (control). When $\beta$-LG is present during heating, the standard curve of PG-594 heated in the presence of $0.5 \% \beta$-LG must be used to characterize PG-594 in heated whey fractions. For PG-594 in heated micelle fractions, the standard curve of PG-594 heated in the presence of $0.5 \% \beta$-LG and casein micelles must be used.

The fluorescent-based concentration assay required sample sizes as small as $100 \mu \mathrm{L}$. The only sample treatment necessary was the addition of trisodium citrate to a final concentration of $0.1 M$ to dissociate micelles and reduce sample turbidity. Fluorescent measurement of the clarified samples took less than $2 \mathrm{~min}$. When performed in a microplate format, high sample throughput can be achieved. These advantages, combined with the demonstrated stability of the PG-594 to common dairy processes as well as the ability to quantify PG in presence of both whey and casein proteins, make the fluorescent labeling approach used in this study attractive for investigating effects of processing treatments on location and concentrations of plasmin system components.

\section{Effect of Heat and $\beta$-LG on Location and Concentration of Labeled PG}

Labeled PG-594 (2.8 $\mu \mathrm{g} / \mathrm{mL})$ was added to simulated milk samples to study the effect of heat and $\beta$-LG on the location and concentration of PG-594. Distribution of PG-594 was analyzed by fluorescent measurement of both whey and micelle fractions, and results were converted to PG-594 concentrations using the appropriate standard curve (shown in Figure 3). This approach was able to recover 97 to 101\% of PG-594 added to the simulated milk system, compared with activitybased assays, which were only capable of recovering $50 \%$ of the PL activity added into milk samples (Choi et al., 2004), presumably due to sample loss during multiple steps of sample preparation.

Before heating, the majority of PG-594 (68.1 $\pm 0.6 \%)$ was associated with micelles (Figure 4). Addition of $\beta$ LG shifted that distribution toward the whey fraction. This agrees well with the distribution pattern of PG- 


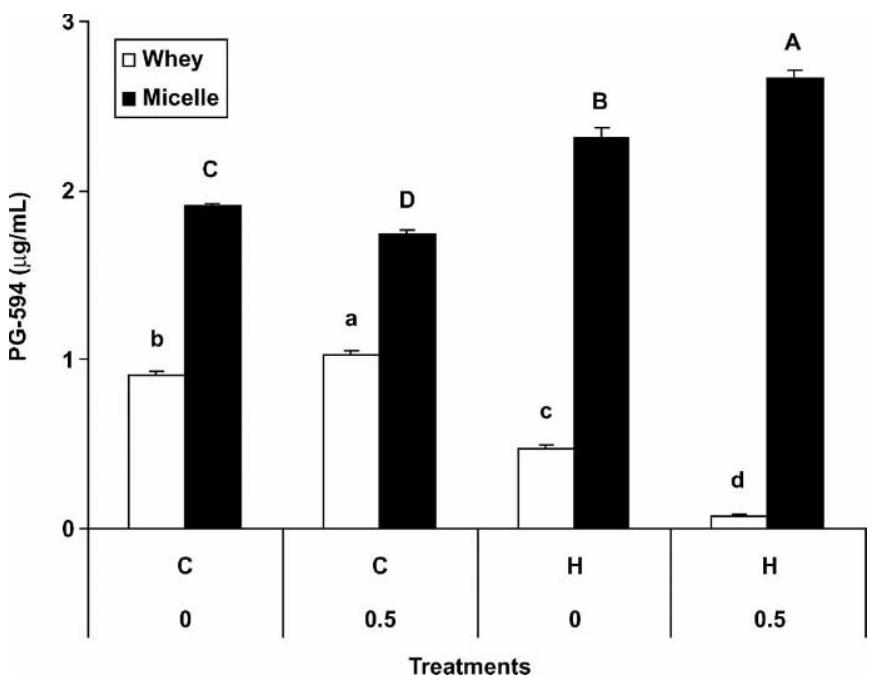

Figure 4. The effects of heat treatment $\left(\mathrm{C}=\right.$ unheated; $\mathrm{H}=85^{\circ} \mathrm{C}$ for $16 \mathrm{~s})$ and $\beta$-LG level $(0$ and $0.5 \%)$ on Alexa Fluor 594 conjugated plasminogen (PG-594) distribution between whey and micelle fractions of simulated bovine milk systems. Values are means of 6 determinations, with error bars representing standard deviation. Different letters above bars indicate significant difference among the means by Duncan multi-range comparison $(P<0.05)$.

derived activities in milk and model systems (Politis et al., 1992). Once heated, both the location and concentration of PG-594 were greatly altered. Activity-based studies on heated samples, which showed a significant reduction of PG-derived activity in both whey and micelle fractions, were only able to detect up to $20 \%$ of the added PG (Sombers and Hayes, 2005). However, the fluorescent-based concentration studies effectively recovered the majority of the added PG (Figure 4). Results showed a shift in distribution of PG-594 toward the micelle. In whey-protein-free systems, heat-induced PG binding to micelles was evidenced by a more than $50 \%$ reduction of PG-594 in whey fractions (from 31 to $15 \%$ ) and a concurrent $20 \%$ increase in micelle fractions (from 69 to 85\%; Figure 4). Presence of $\beta$-LG in heated samples resulted in depletion of PG-594 in the whey fraction and recovery of over 95\% of the total PG-594 in the micelle fractions (Figure 4). Studies have shown that heated $\beta$-LG interacts with casein micelles (Corredig and Dalgleish, 1999; Lowe et al., 2004; Nabhan et al., 2004) and PL (Grufferty and Fox, 1986) through thiol-disulfide exchange. Sodium dodecyl sulfate-PAGE analysis on whey and micelle samples also showed $\beta$ LG covalently binding to micelles in a heat-induced manner (Figure 5). Bicinchoninic acid assay revealed that almost $70 \%$ of $\beta$-LG was adhered to micelles after heating (data not shown). The fact that, when heated, over $90 \%$ of PG-594 was removed from the whey when most of the $\beta$-LG was bound to micelles indicated that

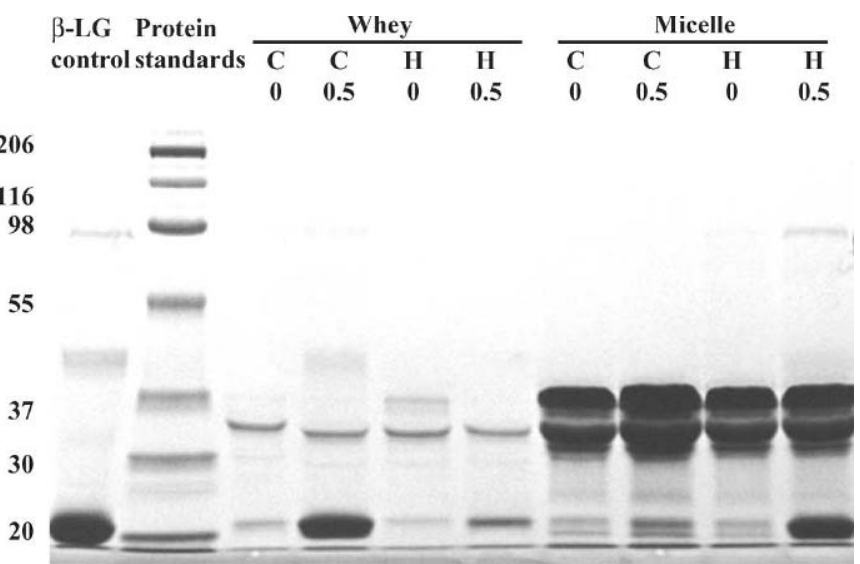

Figure 5. Protein profile of whey and micelle fractions affected by heat treatment $\left(\mathrm{C}=\right.$ unheated; $\mathrm{H}=85^{\circ} \mathrm{C}$ for $\left.16 \mathrm{~s}\right)$ and $\beta$-LG level $(0$ or $0.5 \%)$. Migration of protein standards and pure $\beta$-LG are also shown.

PG-594 might have associated with $\beta$-LG in milk serum and coadhered to micelles during heating.

In Figure 6, the pie diagram of the ratio between type III sum of squares and degree of freedom of the significant factors $(P<0.05)$ showed contributions of the factors to the variation in PG-594 concentration in either whey or micelle fractions. Heat treatment was the most significant factor, accounting for $85 \%$ of the changes in PG-594 distribution. $\beta$-Lactoglobulin and $\beta$ LG-heat interaction accounted for the majority of the rest of the PG-594 changes observed, whereas other factors, such as milk and PG-594 sources had little influence on PG-594 distribution.

Different milk fractionation methods could have an influence on PG-594 distribution. Therefore, acid precipitation as well as ultracentrifugation was used to separate whey and micelle fractions. Results showed that the PG-594 distribution pattern and effects of heat and $\beta$-LG on the PG-594 distribution were the same for both acid precipitation and ultracentrifugation (data not shown).

\section{PG Association with the Casein Micelles}

Because the majority of PG-594 was found to be associated with casein micelles and heating increased PG594 association with the micelles, the mechanism of the association was further analyzed. Many studies have shown that association of native PG to micellar casein involves lysine binding sites, and incubation with EACA at concentrations up to $0.1 M$ can dissociate PG from micelles (Rollema et al., 1983; Baer et al., 1994). In accordance with the above studies, fluorescent intensity measurement (Table 1) showed that EACA was able to 


\section{A. Whey fractions}

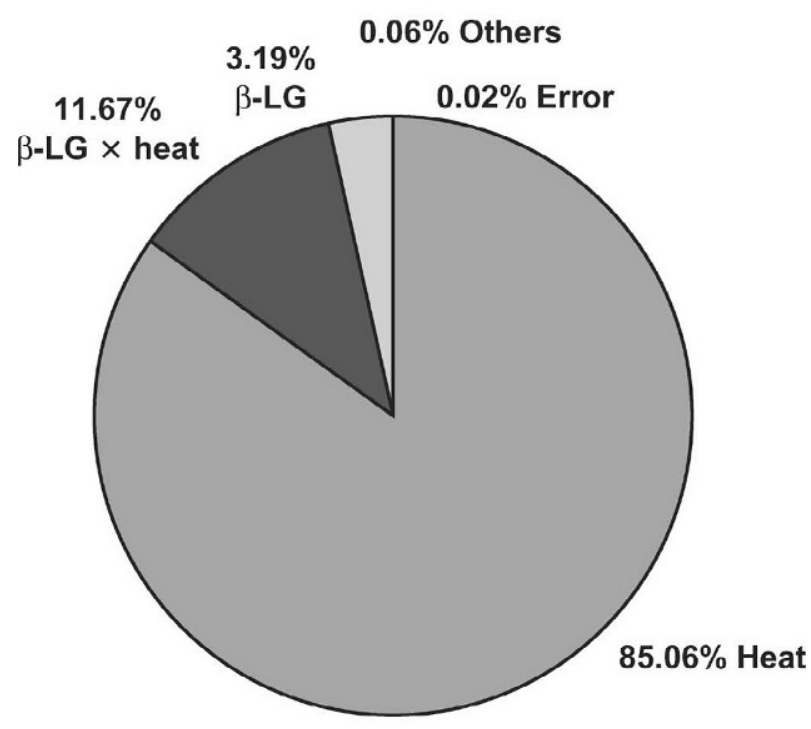

\section{B. Micelle fractions}

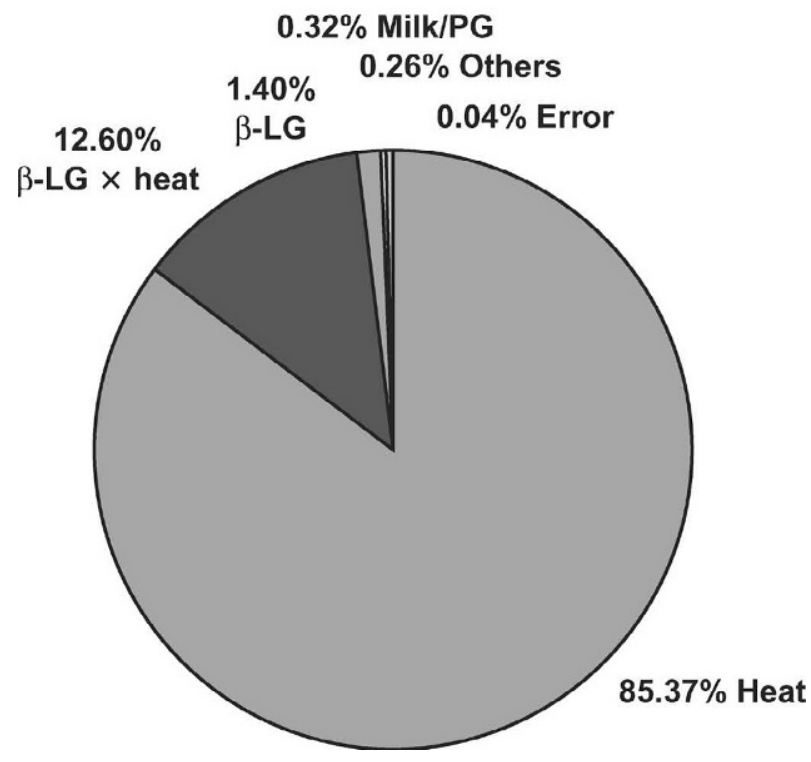

Figure 6. Contributions of the significant factors $(P<0.05)$ to the variability of Alexa Fluor 594 conjugated plasminogen (PG-594) levels in whey (A) and micelle (B) fractions of simulated bovine milk systems. No heat treatment or $85^{\circ} \mathrm{C}$ for $16 \mathrm{~s}, \beta$-LG at 0 or $0.5 \%$, and 3 milk sources that supply fresh casein micelles were examined in a $2 \times 2 \times 3$ factorial model. The ratio of type III sum of squares vs. degree of freedom of each factor is plotted. Heat, $\beta-\mathrm{LG}$, and the interaction of heat and $\beta$-LG were the most significant factors influencing PG594 distributions. Other insignificant factors examined include milk/ micelle source (whey fraction only) and 2-way and 3-way interactions involving milk/micelle source. dissociate more than 70\% of PG-594 from the surface of the micelle in unheated samples. However, once heated, only $23 \%$ dissociation was observed, which further dropped to $4 \%$ when $\beta$-LG was present (Table 1).

The low recovery rate of PG-594 by EACA explains the low activity level detected in the heated micelle fraction because activity-based studies solely depend on the action of EACA to remove casein inhibition and recover micellar PG for activity assessment of heat and $\beta$-LG effects. Therefore, the availability of the remaining bound $\mathrm{PG}$ and its activation by PA deserved further investigation.

The failure to recover more PG-594 from casein micelles using EACA indicated that other interactions independent of lysine binding sites were involved. Studies have demonstrated that the thiol-disulfide exchange occurs between $\beta$-LG and PL/PG upon heating and results in PL inhibition (Grufferty and Fox, 1986; Rollema and Poll, 1986). Moreover, disulfide bonds formed between PG and $\beta$-LG may be responsible for retaining PG on the $\beta$-LG-micelle complex. To test this hypothesis, $2 \mathrm{ME}$ was added to heated micelles and samples were ultracentrifuged to recover dissociated PG-594. About $36 \pm 3.5 \%$ of total PG-594 was recovered by $2 \mathrm{ME}$, demonstrating that disulfide bonding is indeed a major mechanism of PG binding to $\beta$-LG associated with micelles. The remaining $60 \%$ of PG may also be covalently bound. Unlike acid precipitation (in which $0.1 M$ trisodium citrate was added to dissociate casein micelles), use of ultracentrifugation does not disrupt aggregated micelles or micelle- $\beta$-LG complexes formed because of heating. Thus, action of $2 \mathrm{ME}$ might be less effective in recovering $P G$ that is trapped inside of those protein complexes. Nevertheless, if covalent bonds were involved in heat-induced PG association with micelles, PG would likely be unavailable for conversion to PL by PA.

Surprisingly, about $25.6 \pm 7.7 \%$ of PG-594 dissociation by $2 \mathrm{ME}$ (Table 1) was observed in heated $\beta$-LGfree casein micelle samples. Although differences in micelles collected at different times of the year and different stages of lactation over the length of the project could contribute to a large variation of the degree of PG dissociation by $2 \mathrm{ME}$, the effect of $2 \mathrm{ME}$ was significant. The role of casein was previously believed to protect PG denaturation during heating by lysine binding (Grufferty and Fox, 1988b; Metwalli et al., 1998), and covalent interactions between PG and the casein micelle have not been previously reported. Our results suggest that, in addition to lysine bonds, disulfide bonding was one of the major mechanisms of heat-induced PG association with casein. Heat induced disulfide bond redistribution/exchange extensively exists among all sulfite-containing proteins (Visschers and de Jongh, 
Table 1. Disassociation of Alexa Fluor 594-conjugated plasminogen (PG-594) from casein micelles by $0.25 M$ epsilon-aminocaproic acid (EACA) and $0.48 \mathrm{M} \beta$-mercaptoethanol (2ME) showing as percentage of fluorescent intensity of total PG-594 added to milk model system. Values are means of 2 determinations, followed by standard error

\begin{tabular}{|c|c|c|c|c|}
\hline & \multicolumn{4}{|c|}{ Treatment $^{1}$} \\
\hline & \multicolumn{2}{|c|}{ Unheated } & \multicolumn{2}{|c|}{$85^{\circ} \mathrm{C} 16 \mathrm{~s}$} \\
\hline & $0 \% \beta-\mathrm{LG}$ & $0.5 \% \beta$-LG & $0 \% \beta$-LG & $0.5 \% \beta-\mathrm{LG}$ \\
\hline $\begin{array}{l}\text { Micelle total }^{2} \\
\text { EACA dissociation } \\
\text { 2ME dissociation }\end{array}$ & $\begin{array}{l}69.6 \pm 2.2 \\
50.5 \pm 4.4 \\
-\end{array}$ & $\begin{array}{l}65.1 \pm 2.6 \\
46.5 \pm 4.1 \\
-\end{array}$ & $\begin{array}{l}85.3 \pm 1.7 \\
19.7 \pm 2.3 \\
25.7 \pm 7.7\end{array}$ & $\begin{array}{r}96.2 \pm 0.7 \\
3.9 \pm 3.5 \\
35.8 \pm 4.3 \\
\end{array}$ \\
\hline
\end{tabular}

\footnotetext{
${ }^{1}$ Milk model systems were subjected to heat and $\beta$-LG treatments, and the resulting casein micelle fractions were analyzed for PG-594 bonding.

${ }^{2}$ Recovered by ultracentrifugation.

${ }^{3}$ Recovered by acid precipitation.
}

2005). The disulfide interaction between $P G$ and sulfite containing caseins, mainly $\alpha_{\mathrm{S} 1^{-}}$and $\kappa$-casein, may be responsible for reduced activity observed in $\beta$-LG-free micelle systems. Metwalli et al. (1998) proposed a reaction scheme to illustrate inactivation of plasmin in heated milk, in which irreversible PL inactivation in the absence of $\beta$-LG occurs mainly via irreversible unfolding. Our results suggest disulfide linkages between $\mathrm{PG}$ and caseins appear to be the main mechanism for $\mathrm{PG}$ inactivation in $\beta$-LG-free systems.

\section{Western Blotting and Zymography}

To compare fluorescent measurement results with an existing quantitative analysis method, micelle samples were analyzed on Western blots. In addition, caseinuPA zymography was used to analyze active PG in micelle fractions (Figure 7). However, compared with the fluorescent technique, which only measures the added
PG-594, Western blots and zymography measure both intrinsic native PG and added PG-594. Additionally, due to casein inhibition, only PG that is disassociated from micelles was visible. On both zymography and Western blots, dissociated PG showed as a $\sim 100 \mathrm{kDa}$ band under nonreducing conditions but migrated slightly more slowly under reducing conditions and appeared as a $\sim 105 \mathrm{kDa}$ band (Figure 7).

Lysine-bound PG can be dissociated from micelles by EACA treatment before electrophoresis. Additionally, PG and micelle association involving lysine binding sites was likely to be disrupted under electrophoresis conditions. Therefore, the electrophoresis control samples showed the same reaction patterns as the EACAtreated samples on both zymography and Western blots. The casein-uPA zymography revealed the lysis zones due to activation of $\mathrm{PG}$ dissociated from micelles. As expected, the size of the lysis zones was strongly affected by heat treatment: unheated samples gave rise

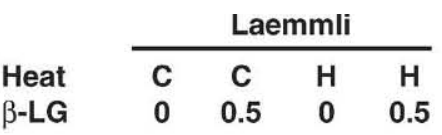

\begin{tabular}{cccc}
\multicolumn{4}{c}{ Laemmli + EACA } \\
\hline C & C & H & H \\
0 & 0.5 & 0 & 0.5
\end{tabular}

\begin{tabular}{cccc}
\multicolumn{4}{l}{ Laemmli + } \\
\hline C & C & H & H heat \\
0 & 0.5 & 0 & 0.5
\end{tabular}

Casein-uPA
Zymography

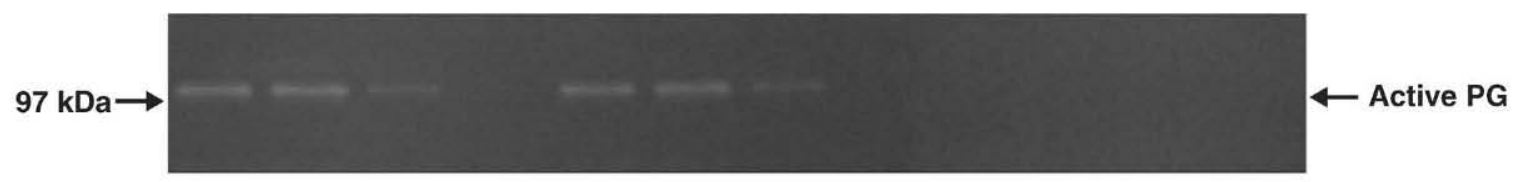

Anti-PG

Western

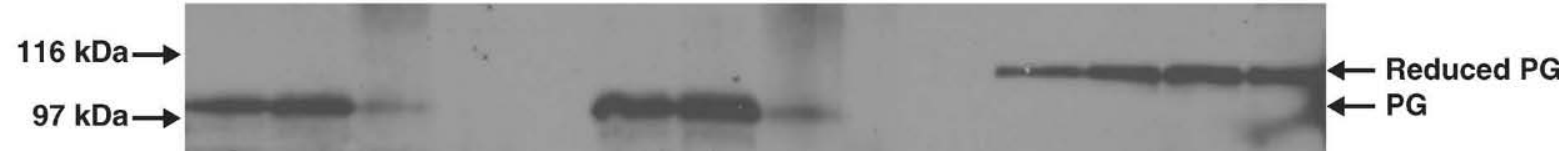

Figure 7. Visualization of plasminogen (PG) in micelle fractions affected by heat treatment $\left(\mathrm{C}=\right.$ unheated; $\mathrm{H}=85^{\circ} \mathrm{C}$ for $\left.16 \mathrm{~s}\right)$ and $\beta$ LG level (0 or 0.5\%). Visualized PG includes both intrinsic native PG and added Alexa Fluor 594 conjugated plasminogen (PG-594). Plasminogen was dissociated from micelles by epsilon-aminocaproic acid (EACA), $\beta$-mercaptoethanol (2ME), and during electrophoresis. Lysis zones of activated PG are visualized on casein-urokinase zymography and PG quantities are detected by anti-PG Western blots. Migration of protein standards is indicated on the right and that of PG control on the left. 
to the largest lysis zones, heated samples gave rise to smaller lysis zones, and $\beta$-LG inhibited protease activity in heated samples (Figure 7). The immunoreactive PG was similarly affected by heat treatment and $\beta$-LG. The resemblance of the PG patterns on both zymography and Western blots indicated that the PG dissociated from micelles under nonreducing conditions could be activated by uPA and form a lysis zone on the casein gel. These results agree well with fluorescent measurements reported earlier in this study and activity measurements reported by Sombers and Hayes (2005). Activity results of micellar PG greatly depend upon the ability of EACA to dissociate lysine-bound PG from micelles. The consistency between fluorescent measurement and activity measurement on PG distribution indicated that $\mathrm{PG}$ was mainly associated with micelles by lysine bonds. The fact that $\beta$-LG had little effect on this distribution reflects that there is no specific interaction between $\mathrm{PG}$ and $\beta$-LG in raw milk. It also indicates that the reduction of lysis zones in heated samples was mainly due to inefficient release of $\mathrm{PG}$ from micelles.

In contrast, when reducing conditions were applied to disrupt disulfide bonds, a significant amount of PG was detected in heated samples on the Western blot. Although overall signal level was lower than nonreduced samples because of lower affinity of antibody to reduced $\mathrm{PG}$, an increase in $\mathrm{PG}$ intensity in heated samples was obvious. Under similar conditions, $\mathrm{PG}$ is denatured and unable to give rise to active PL; therefore, no lysis zone was observed on casein gels.

Plasmin activity present on the casein-uPA zymogram gel was minimal. Thus, $\mathrm{PG}$ activation during sample treatment was insignificant and all fluorescent measurement truly reflects the behavior of PG-594 rather than PG-594-derived PL, which would have the same fluorescent properties as PG-594 (data not shown). The high PG/PL ratio also verifies that the action of PA was very limited, probably due to PA inactivation by $\beta$-LG during heating (Saint Denis et al., 2001b).

There is native unlabeled PG present in the samples that could affect results. However, both activities visualized on casein-uPA gels and the quantity of PG observed on Western blots correlate well with results from the activity assay and the fluorescent labeling assay on the micellar fraction of PG test samples. The effects of EACA and 2ME on both activity and concentration of PG-594 support the idea that heat-induced disulfide bonding between PG and casein as well as the broadly recognized disulfide bonding between $\mathrm{PG}$ and $\beta$-LG are responsible for $\mathrm{PG}$ association with casein micelles and PG inhibition.

\section{Confocal Microscopy}

Fluorescent confocal microscopy was used to visualize the distribution of fluorescent PG-594 in simulated milk systems. The projections of the unfractionated samples $(25 \mu \mathrm{m}$ thick $\times 160 \mu \mathrm{m} \times 160 \mu \mathrm{m})$ are shown in Figure 8. The distribution of PG-594 observed by fluorescent microscopy agreed well with spectrofluorometry measurement on fractionated PG test samples. The PG-594 associated with micelles or micelle clusters appeared as bright clouds or cloud dust, whereas soluble PG594 (whey fraction) gave rise to the background glow. Addition of $\beta$-LG had little effect on the distribution of PG-594 and the structure of micelles or micelle clusters in unheated samples. Once heated, a dramatic reduction in background brightness was obvious, indicating a decrease of PG-594 concentration in the whey. At the same time, micelle clusters appeared brighter and denser. Presence of $\beta$-LG during heating further reduced the background brightness. Due to disulfide bond formation, the fluorescence intensity of $\beta$-LG-PG-594 was also lowered. On the other hand, the dense structure of micelle aggregates formed upon heating appeared to be unaffected by addition of $\beta$-LG. Heat most likely induced micelle-micelle aggregations, in addition to PG associations to micelles. Anema and $\mathrm{Li}$ (2003) showed that increases in casein micelle size during heating were mainly due to association of $\beta$-LG with the micelles. The images in Figure 8 suggest it is also possible that aggregation of casein micelles could account for the changes in size observed by Anema and Li.

\section{Protein-Protein Interaction Model}

Based on the above results, a molecular model is proposed to illustrate interactions between milk proteins and PG. In a milk model system, the majority of PG binds to casein micelles. There is equilibrium between free PG in whey and bound PG on micelles, probably controlled by availability of lysine binding sites on micelles (Haissat et al., 1994) and rate of association and dissociation of lysine bonds (Markus et al., 1978). This type of interaction can be easily disrupted by addition of EACA. In unheated milk samples, $\beta$-LG has no specific interaction with PG and thus has little effect on its distribution. When heat is applied, half of the free PG becomes associated with micelles and new disulfide bonds start to form between $\kappa$ - (or $\alpha$-) casein and PG. Because of such covalent modification to PG, PG-derived activity decreases despite the increase in the total amount of PG molecules bound to micelles. Because $\beta$-LG contains a free thiol group, it readily forms new disulfide bonds with itself or other proteins, such as PG and casein, when heat-treated. The result is formation of a casein-PG- $\beta$-LG complex; and nearly 

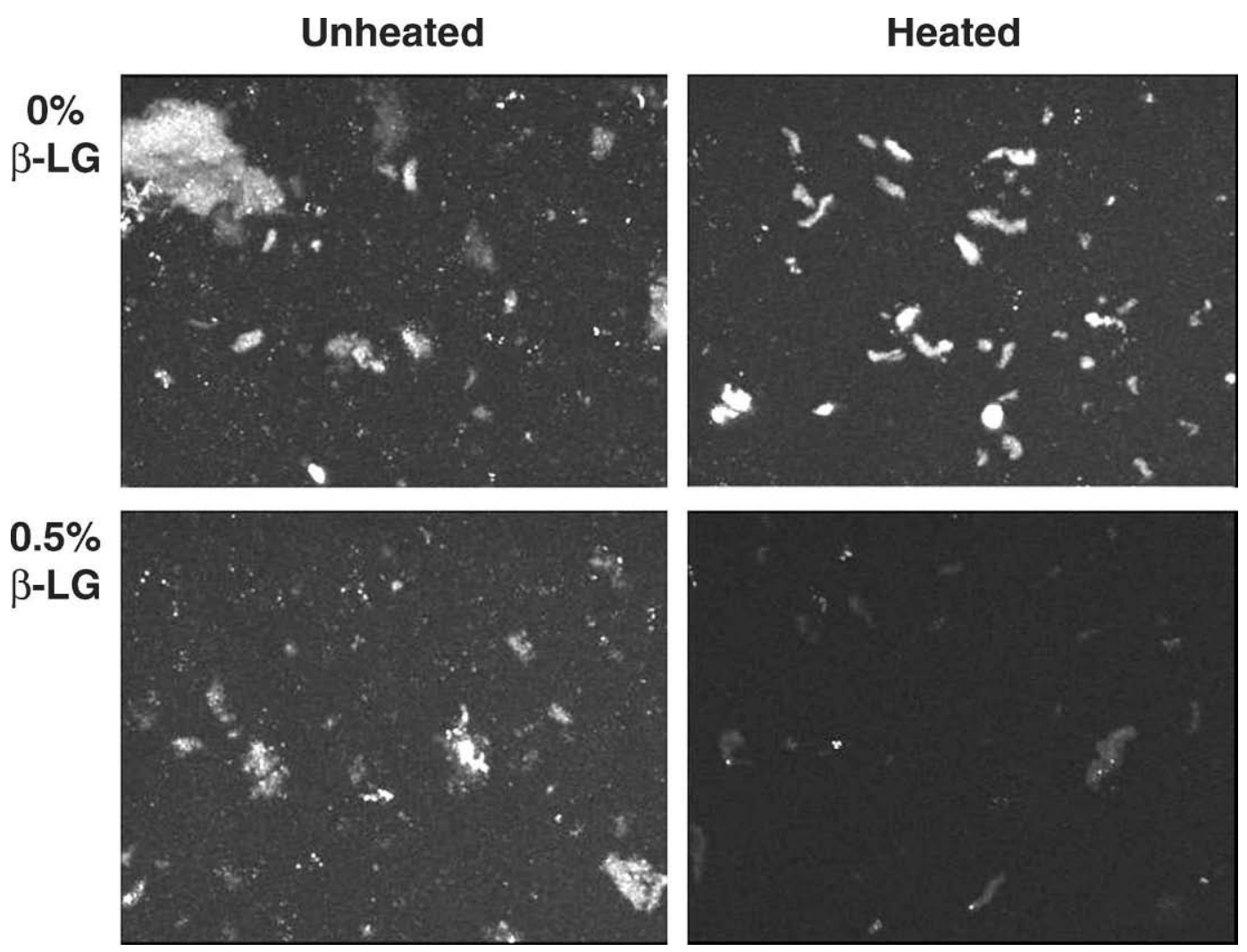

Figure 8. Confocal fluorescent images of distribution of Alexa Fluor 594 conjugated plasminogen (PG-594) in unfractionated milk model systems. Effects of heat treatment (unheated vs. heated at $85^{\circ} \mathrm{C}$ for $\left.16 \mathrm{~s}\right)$ and $\beta$-LG level $(0$ or $0.5 \%)$ on PG-594 distribution were examined. Magnification was $\times 60$.

all of the PG becomes covalently attached to this complex and loses its activity.

\section{CONCLUSIONS}

The fluorescent-labeling method developed was found to be a useful method for measuring the concentration and location of plasminogen, one of the plasmin system components. The distinctive advantages of the fluorescent method are that it can perform a measurement within minutes with simple preparation procedures, it can quantify PG in the presence of whey and casein proteins, and activity studies can be conducted using the same samples to gather information about concentration and activity. Results demonstrated broad applicability of the fluorescent labeling technique as a research tool for characterization of plasmin system components in dairy products and processes.

In combination with fractionation techniques, the fluorescent labeling method was used to successfully characterize PG location in milk systems affected by heating. A differential distribution pattern of PG was found, and effects of heat treatment on the distribution pattern indicated that PG binds to micelles in a heat- induced manner. Incubation with $2 \mathrm{ME}$ to dissociate $\mathrm{PG}$ from micelles revealed that the formation of disulfide bonds between PG and casein as well as between PG and $\beta$-LG was responsible for higher levels of PG in casein fractions. This information, as well as the fluorescent method to quantify PG in the presence of casein and whey proteins, will increase the understanding of PG concentration and location in dairy products.

\section{ACKNOWLEDGMENTS}

This research was supported by USDA-NRICGP Grant \# 04-35503-14129. This is Journal Article no. 2005-17744 of the Purdue University Agricultural Research Programs. We sincerely thank Jennie Sturgis at Purdue University Cytometry laboratories for assistance in preparing confocal microscopy data, Bill Aimutis for general advice, and Nilupa Gunaratna for support in statistical analysis.

\section{REFERENCES}

Alichanidis, E., J. H. M. Wrathall, and A. T. Andrews. 1986. Heatstability of plasmin (milk proteinase) and plasminogen. J. Dairy Res. 53:259-269. 
Anema, S. G., and Y. Li. 2003. Association of denatured whey proteins with casein micelles in heated reconstituted skim milk and its effect on casein micelle size. J. Dairy Res. 70:73-83.

Baer, A., I. Ryba, and J. C. Collin. 1994. Binding of bovine plasminogen to immobilized casein and its activation thereon. Int. Dairy J. 4:597-616.

Bastian, E. D., and R. J. Brown. 1996. Plasmin in milk and dairy products: An update. Int. Dairy J. 6:435-457.

Bastian, E. D., R. J. Brown, and C. A. Ernstrom. 1991. Casein interference in bovine plasmin assays using a synthetic substrate. J. Dairy Sci. 74:4119-4124.

Bastian, E. D., K. G. Hansen, and R. J. Brown. 1993. Inhibition of plasmin by beta-lactoglobulin using casein and a synthetic substrate. J. Dairy Sci. 76:3354-3361.

Benfeldt, C., L. B. Larsen, J. T. Rasmussen, P. A. Andreasen, and T. E. Petersen. 1995. Isolation and characterization of plasminogen and plasmin from bovine-milk. Int. Dairy J. 5:577-592.

Benfeldt, C., J. Sorensen, K. H. Ellegard, and T. E. Petersen. 1997. Heat treatment of cheese milk: Effect on plasmin activity and proteolysis during cheese ripening. Int. Dairy J. 7:723-731.

Centonze, V. E., M. Sun, A. Masuda, H. Gerritsen, and B. Herman. 2003. Fluorescence resonance energy transfer imaging microscopy. Biophotonics A 360:542-560.

Chamberlain, C., and K. M. Hahn. 2000. Watching proteins in the wild: Fluorescence methods to study protein dynamics in living cells. Traffic 1:755-762.

Choi, L. H., A. L. Kelly, S. S. Nielsen, and K. D. Hayes. 2004. Comparison of methods to measure various levels of plasmin in milk. IFT Annu. Mtg. Expo., Las Vegas, NV.

Coons, A. H. 1951. Fluorescent antibodies as histochemical tools. Fed. Proc. 10:558-559.

Corredig, M., and D. G. Dalgleish. 1999. The mechanisms of the heatinduced interaction of whey proteins with casein micelles in milk. Int. Dairy J. 9:233-236.

Crudden, A., and A. L. Kelly. 2003. Studies of plasmin activity in whey. Int. Dairy J. 13:987-993.

de Wit, J. N. 1998. Nutritional and functional characteristics of whey proteins in food products. J. Dairy Sci. 81:597-608.

Derham, O., and A. T. Andrews. 1982. The roles of native milk proteinase and its zymogen during proteolysis in normal bovine-milk. J. Dairy Res. 49:577-585.

Dufour, E., and A. Riaublanc. 1997. Potentiality of spectroscopic methods for the characterisation of dairy products. I. Front-face fluorescence study of raw, heated and homogenised milks. Lait 77:657-670.

Dupont, D., and R. Grappin. 1998. ELISA for differential quantitation of plasmin and plasminogen in cheese. J. Dairy Res. 65:643-651.

Dupont, D., B. Remond, and J. C. Collin. 1998. ELISA determination of plasmin and plasminogen in milk of individual cows managed without the dry period. Milchwissenschaft 53:66-69.

Enright, E., A. P. Bland, E. C. Needs, and A. L. Kelly. 1999. Proteolysis and physicochemical changes in milk on storage as affected by UHT treatment, plasmin activity and KIO3 addition. Int. Dairy J. 9:581-591.

Enright, E., and A. L. Kelly. 1999. The influence of heat treatment of milk on susceptibility of casein to proteolytic attack by plasmin. Milchwissenschaft 54:491-493.

Fajardo-Lira, C., M. Oria, K. D. Hayes, and S. S. Nielsen. 2000. Effect of psychrotrophic bacteria and of an isolated protease from Pseudomonas fluorescens M3/6 on the plasmin system of fresh milk. J. Dairy Sci. 83:2190-2199.

Fox, P. F. 1989. Proteolysis during cheese manufacturing and ripening. J. Dairy Sci. 72:1379-1400.

Fox, P. F., and P. L. H. McSweeney. 1996. Proteolysis in cheese during ripening. Food Rev. Int. 12:457-509.

Fox, P. F., and P. L. H. McSweeney, ed. 2003. Advanced Dairy Chemistry Vol. 1 Proteins. 3rd ed. Kluwer Academic/Plenum Publisher, New York, NY.

Grufferty, M. B., and P. F. Fox. 1986. Potassium iodate-induced proteolysis in ultra heat-treated milk during storage-The role of betalactoglobulin and plasmin. J. Dairy Res. 53:601-613.
Grufferty, M. B., and P. F. Fox. 1988a. Milk alkaline proteinase. J. Dairy Res. 55:609-630.

Grufferty, M. B., and P. F. Fox. 1988b. Heat-stability of the plasmin system in milk and casein systems. N.Z. J. Dairy Sci. Technol. 23:143-152.

Haissat, S., E. Marchal, P. Montagne, G. Humbert, M. C. Bene, G. Faure, and G. Linden. 1994. Quantitative characterization of bovine plasminogen binding to caseins. Anal. Biochem. 222:472-478.

Hayes, M. G., P. L. H. McSweeney, and A. L. Kelly. 2002. The influence of native and heat-denatured whey proteins on enzyme activity. 1. Plasmin. Milchwissenschaft 57:208-211.

Herman, B., R. V. Krishnan, and V. E. Centonze. 2004. Microscopic analysis of flourescence resonance energy transfer (FRET). Pages 351-370 in Protein-Protein Interactions, Methods and Applications. H. Fu, ed. Humana Press, Totowa, NY.

Holmes, K. L., and L. M. Lantz. 2001. Protein labeling with fluorescent probes. Methods Cell Biol. 63:185-204.

Jenness, R., and J. Koops. 1962. Preparation and properties of a salt solution which simulates milk ultrafiltrate. Neth. Milk Dairy J. 16:153-163.

Kelly, A. L. 1999. The influence of heat treatment of milk on proteolysis in cheddar cheese during ripening. Milchwissenschaft 54:682-685.

Kenworthy, A. K. 2001. Imaging protein-protein interactions using fluorescence resonance energy transfer microscopy. Methods 24:289-296.

Korycka-Dahl, M., B. R. Dumas, N. Chene, and J. Martal. 1983. Plasmin activity in milk. J. Dairy Sci. 66:704-711.

Kulmyrzaev, A., and E. Dufour. 2002. Determination of lactulose and furosine in milk using front-face fluorescence spectroscopy. Lait 82:725-735.

Lakowicz, J. R. 1999. Principles of fluorescence spectroscopy. 2nd ed. Kluwer Academic/Plenum Publisher, New York, NY.

Lowe, E. K., S. G. Anema, A. Bienvenue, M. J. Boland, L. K. Creamer, and R. Jimenez-Flores. 2004. Heat-induced redistribution of disulfide bonds in milk proteins. 2. Disulfide bonding patterns between bovine beta-lactoglobulin and kappa-casein. J. Agric. Food Chem. 52:7669-7680.

Lu, D. D., and S. S. Nielsen. 1993. Assays for native plasminogen activators in bovine-milk. J. Dairy Sci. 76:3362-3368.

Markus, G., J. L. Depasquale, and F. C. Wissler. 1978. Quantitativedetermination of binding of epsilon-aminocaproic acid to native plasminogen. J. Biol. Chem. 253:727-732.

Metwalli, A. A. M., H. H. J. de Jongh, and M. van Boekel. 1998. Heat inactivation of bovine plasmin. Int. Dairy J. 8:47-56.

Miller, L. W., and V. W. Cornish. 2005. Selective chemical labeling of proteins in living cells. Curr. Opin. Chem. Biol. 9:56-61.

Nabhan, M. A., J. M. Girardet, S. Campagna, J. L. Gaillard, and Y. Le Roux. 2004. Isolation and characterization of copolymers of

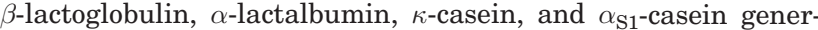
ated by pressurization and thermal treatment of raw milk. J. Dairy Sci. 87:3614-3622.

Nielsen, S. S. 2002. Plasmin system and microbial proteases in milk: Characteristics, roles, and relationship. J. Agric. Food Chem. 50:6628-6634

Patton, W. F. 1999. Proteome analysis II. Protein subcellular redistribution: Linking physiology to genomics via the proteome and separation technologies involved. J. Chromatogr. B Analyt. Technol. Biomed. Life Sci. 722:203-223.

Politis, I., D. M. Barbano, and R. C. Gorewit. 1992. Distribution of plasminogen and plasmin in fractions of bovine milk. J. Dairy Sci. 75:1402-1410.

Politis, I., B. Zavizion, D. M. Barbano, and R. C. Gorewit. 1993. Enzymatic assay for the combined determination of plasmin plus plasminogen in milk-revisited. J. Dairy Sci. 76:1260-1267.

Richardson, B. C., and K. N. Pearce. 1981. The determination of plasmin in dairy-products. N.Z. J. Dairy Sci. Technol. 16:209-220.

Rollema, H. S., and J. K. Poll. 1986. The alkaline milk proteinase system-Kinetics and mechanism of heat-inactivation. Milchwissenschaft 41:536-540. 
Rollema, H. S., S. Visser, and J. K. Poll. 1983. Spectrophotometric assay of plasmin and plasminogen in bovine-milk. Milchwissenschaft 38:214-217.

Saint Denis, T., G. Humbert, and J. L. Gaillard. 2001a. Enzymatic assays for native plasmin, plasminogen and plasminogen activators in bovine milk. J. Dairy Res. 68:437-449.

Saint Denis, T., G. Humbert, and J. L. Gaillard. 2001b. Heat inactivation of native plasmin, plasminogen and plasminogen activators in bovine milk: A revisited study. Lait 81:715-729.

Scollard, P. G., T. P. Beresford, E. C. Needs, P. M. Murphy, and A. L. Kelly. 2000. Plasmin activity, $\beta$-lactoglobulin denaturation and proteolysis in high pressure treated milk. Int. Dairy J. 10:835841.

Sombers, S. E., and K. D. Hayes. 2005. The effects of whey proteins and heat treatment on binding of plasminogen and plasminogen activators to casein micelles in a simulated milk system. First IDF Int. Symp. Indigenous Enzymes in Milk, Univ. Coll. Cork, Ireland.

Swaisgood, H. E. 1996. Characteristics of milk. Marcel Dekker, Inc., New York, NY.

Visschers, R. W., and H. H. J. de Jongh. 2005. Disulphide bond formation in food protein aggregation and gelation. Biotechnol. Adv. 23:75-80. 